\title{
Hepatoid carcinoma of ovary: a very rare histopathological finding
}

\author{
Nahida Hassan Wani ${ }^{1 *}$, Rupinder Sekhon ${ }^{1}$, Shevta Giri ${ }^{1}$, Sudhir K. Rawal ${ }^{2}$
}

\author{
${ }^{1}$ Department of Gyne-Oncology, Rajiv Gandhi Cancer Institute and Research Centre, New Delhi, India \\ ${ }^{2}$ Department of Uro-Gyne-Oncology, Rajiv Gandhi Cancer Institute and Research Centre, New Delhi, India
}

Received: 13 July 2014

Accepted: 20 July 2014

\section{*Correspondence:}

Dr. Nahida Hassan Wani,

E-mail: dr.nahidhassan@gmail.com

(C) 2014 Wani NH et al. This is an open-access article distributed under the terms of the Creative Commons Attribution Non-Commercial License, which permits unrestricted non-commercial use, distribution, and reproduction in any medium, provided the original work is properly cited.

\begin{abstract}
Hepatoid carcinoma of the ovary is a rare histopathological variant that has phenotypic properties in common with hepato-cellular carcinoma and composed mainly of epitheloid cells. It presents with unilateral or bilateral ovarian masses with elevated serum $\alpha$-feta-protein especially in post-menopausal female. We hereby discuss a very rare case of 58 years old female presented with abdominal pain and distension with high serum levels of CA 125 and AFP. She was diagnosed as hepatocellular carcinoma. CT guided FNAC of omentum revealed poorly differentiated carcinoma and on immuno-histochemical staining cells was positive for CK7, CK8/17 and AFP. The tumor cells were negative for CMA, PAX2/PAX8, CK20 and CK19. She was diagnosed with bilateral hepatoid carcinoma of ovary. This shows that histopathological examination and tissue immuno-histochemical staining plays an important role in distinguishing hepatoid variety of ovarian carcinoma from other ovarian tumors and hepatocellular carcinoma.
\end{abstract}

Keywords: $\alpha$-fetoprotein, CA125, Hepatoid carcinoma of ovary, Hepatocellular carcinoma, Immunohistochemistry

\section{INTRODUCTION}

Hepatoid carcinomas are less frequently occurring tumors of the stomach, lungs, kidneys, endometrium and ovaries with pathological features similar to those of hepatocellular carcinoma (HCC). Hepatoid Carcinoma of the Ovary $(\mathrm{HCO})$ is a very rare type of high-grade, invasive malignant ovarian tumor composed mainly of epitheloid cells. Hepatoid carcinoma of ovary was first reported in 1987. This malignancy has been reported mainly in post-menopausal women presenting with unilateral or bilateral ovarian masses and elevated serum level of $\alpha$-fetoprotein (AFP). ${ }^{1}$

Microscopically, these tumors are characterized by solid sheets of large cells with abundant eosinophilic cytoplasm; centrally pleomorphic nuclei with distinctcellular borders. ${ }^{2}$ On IHC cells are positive for CK7 and AFP.

\section{CASE REPORT}

A 58 year old postmenopausal female with no comorbidity presented at our institute as a diagnosed case of metastatic hepatocellular carcinoma elsewhere with chief complaints of abdominal pain and distension and weight loss for one month, with no significant past medical or surgical history and family history. On physical examination, her performance scale was 4 with mild anemia and no lymphadenopathy. On per abdomen gross ascites was present. On P/V/R examination no abnormality was present except for left adnexal mass of size about 4 x $4 \mathrm{~cm}$ hard inconsistency, restricted mobility, separate from the uterus. An increased CA 125 at $820 \mathrm{U} / \mathrm{ml}$ (normal <35) and $\alpha$-fetoprotein $=11000$ $\mathrm{U} / \mathrm{ml}$ (normal 0.5 to 5.5 ) and carcino-embryonic antigen (CEA) - 4.19 (normal 0-3 ng/ml).

CECT abdomen showed left adnexal mass size $4 \times 2.4 \mathrm{x}$ $1.6 \mathrm{~cm}$ with omental caking and gross ascites with 
multiple peritoneal deposits, no liver parenchymal lesion. MRI abdomen showed left adnexal mass, omental thickening with peritoneal nodules. Patient had underwent ascitic tapping along with CT guided FNAC from the omentum elsewhere which showed poorly differentiated carcinoma. For confirmation of diagnosis Tru cut biopsy from the omentum was done at our institute which showed hepatoid cells, on IHC: tumor cells was positive for Heppar-1, Glypican-3, CK8/18, CK $7 \&$ AFP and negative for SALL-4, CK20, PAX-8. Upper and lower GI endoscopy were normal. Since liver and gastrointestinal tract showed no primary growth, along with only an adnexal mass so primary diagnosis of hepatoid carcinoma of ovarian was made. Due to advanced stage of disease and associated increased morbidity with the surgery patient received 3 cycles of neoadjuvant chemotherapy (Carboplatin and paclitaxel) followed by interval-cytoreductive surgery. She underwent interval cytoreductive surgery i.e. total abdominal hysterectomy with bilateral salphingoophorectomy, omentectomy, appendectomy along with bilateral pelvic lymphadenectomy, peritonectony was done and mesenteric deposits were removed. Per operative findings: ascites of about 2-2.5 litres, numerous tumor deposits were seen on bilateral hemi-diaphragm, mesentery of large and small intestine and omental caking was present. Right ovary measures $\sim 2.5$ x 0.8 x $0.6 \mathrm{~cm}$, left ovary measures $\sim 2.4 \times 0.6 \times 0.8 \mathrm{~cm}$ with capsules intact with multiple of grey white nodules present ranging from 0.1 to $2.5 \mathrm{~cm}$ were present.

On microscopic examination: bilateral ovaries showed a poorly differentiated carcinoma having a sinusoidal pattern with cells arranged in multilayered trabeculae with cells having vesicular round nucleus and prominent nucleoli the tumor has surface as well as intraparenchymal growth. On IHC: the tumor cells express CK8/18, CK and AFP, Heppar-1, and Glypican3 , the tumor cells were negative for SALL-4, EMA, PAX-8, CK20 and CK19. Intra-operative and postoperative period was uneventful. After surgery she received 3 cycles adjuvant chemotherapy. The patient is on continuous follow up till date with no recurrence.

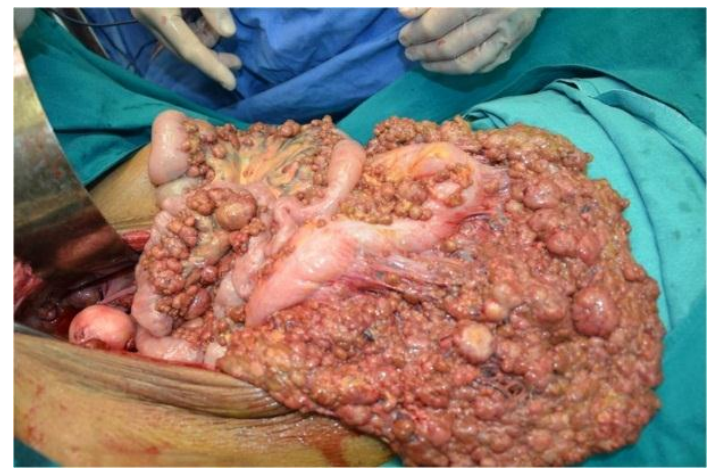

Figure 1: Per operative findings: ascites of about 22.5 litres, numerous tumor deposits were seen on bilateral hemi-diaphragm, mesentery of large and small intestine and omental caking was present.

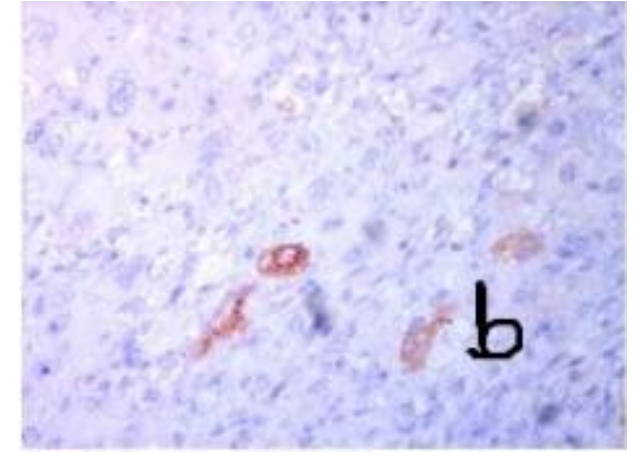

Figure 2: Shows positive cells on IHC for AFP.

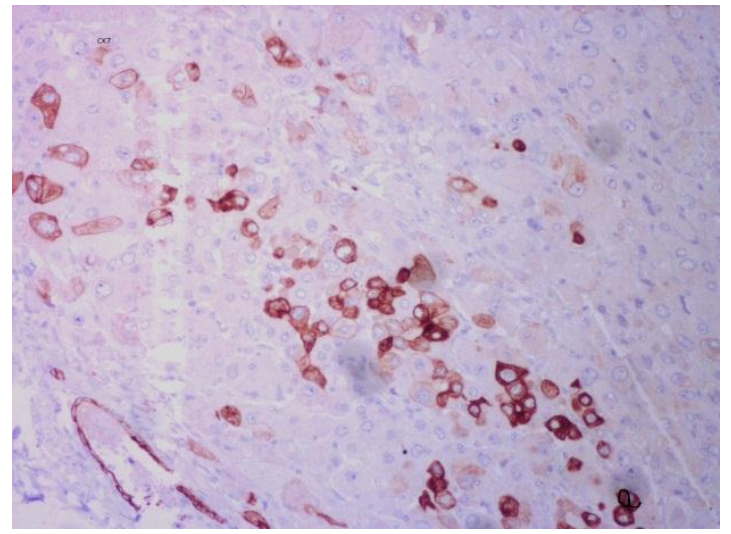

Figure 3: The tumor cells on IHC positive for CK7.

\section{DISCUSSION}

The first case of hepatoid ovarian tumor was described in 1987. Originally HCO a rare ovarian tumor that usually presents with signs and symptoms of an adnexal mass such as progressive abdominal distension and lower abdominal pain, has been described in only postmenopausal women aged from 42 to 78 years (average, 63 years $)^{1}$. This malignancy mostly was found at an advanced clinical stage and progress rapidly, with metastases to the abdomen and occasionally to the lungs; with median survival about 2 years. ${ }^{3}$

Macroscopically, HCOs are solid and cystic. Histologically, $\mathrm{HCO}$ resembles $\mathrm{HCC}^{2}{ }^{2} \mathrm{HCO}$ is associated with elevated serum AFP and CA 125 levels. The CK is highly consistent with $\mathrm{HCO},{ }^{4}$ differs from hepatocytes and resembles common epithelial adenocarcinoma, suggesting epithelial origin of $\mathrm{HCO} .^{5}$

In our case tumor cells express Glypican-3, AFP, CK7, $\mathrm{P} 53$ and CK8/18, on IHC staining and were negative for EMA and PAX-2/PAX-Q. Based on aforementioned findings, final diagnosis of hepatoid variety of ovarian carcinoma was made.

Differential diagnosis of HCO includes yolk sac tumors of the ovary and endometrial carcinoma, clear cell carcinoma and undifferentiated carcinoma. ${ }^{1}$ Yolk sac tumors of the ovary are rare but highly likely to be 
malignant and increase rapidly in size. They express high amounts of AFP, which increases with tumor progression. In the clinical setting, the presence of the globules, along with the production of AFP, can be diagnosed as yolk sac tumors of the ovary. ${ }^{6}$ Endometrial adenocarcinomas rarely produce AFP. Microscopically, endometrial tumors are usually composed of a major medullary portion and a minor tubular adenocarcinoma invading the myometrium. Vascular permeation by neoplastic cells are prominent and extensive hepatoma like features are often observed. ${ }^{7}$

To date, there is insufficient data regarding the optimal treatment of patients with HCO. Most patients are treated by surgery, followed by a chemotherapy regimen similar to those used in patients with ovarian-like carcinoma. HCOs are highly aggressive with $75 \%$ of patients with stage III or IV diseases. In our case the treatment of choice was neoadjuvant chemotherapy followed by interval debulking along with adjuvant chemotherapy for 3 cycles. Follow-up of the patient showed no evidence of tumor recurrence or ascites after initial diagnosis.

\section{CONCLUSIONS}

Hepatoid carcinoma of ovary is a rare histopathological variant that has phenotypic properties in common with hepatocellular carcinoma and till now 20 cases have been reported. Patient with $\mathrm{HCO}$ should be managed same as that of epithelial carcinoma of ovary. Hepatoid carcinoma of ovary and metastatic hepatoid carcinoma from other organs share numerous clinicopathological features such as elevated AFP serum levels, hepatoid morphology. Immuno-histochemical staining plays an important role for confirmation of diagnosis. Therefore it is advised, before considering primary hepatoid carcinoma of ovary, the liver and GI tract should be excluded.
Funding: No funding sources Conflict of interest: None declared

Ethical approval: Not required

\section{REFERENCES}

1. Ishikura H, Scully RE. Hepatoid carcinoma of the ovary: a newly described tumor. Cancer. 1987;60:2775-84.

2. Zizi-Sermpetzoglou A, Petrakopoulou N, Nikolaidou ME, Tepelenis N, Savvaidou V, Vasilakaki T. Hepatoid carcinoma of the ovary: a case report and review of the literature. Eur $\mathbf{J}$ Gynaecol Oncol. 2009;30:341-3.

3. Lazaro J, Rubio D, Repolles M, Capote L. Hepatoid carcinoma of the ovary and management. Acta Obstet Gynecol Scand. 2007;86:498-9.

4. Pandey M, Truica C. Hepatoid carcinoma of the ovary. J Clin Oncol. 2011;29(15):e446-8.

5. Tochigi N, Kishimoto T, Supriatna Y, Nagai Y, Nikaido T, Ishikura H. Hepatoid carcinoma of the ovary: a report of three cases admixed with a common surface epithelial carcinoma. Int J Gynecol Pathol. 2003;22:266-71.

6. Beurdeley M, Gauthier T, Piquet C, Fourcade L. Conservative treatment of big yolk sac tumor of the ovary in young girl. J Visc Surg. 2010;147:e265-7.

7. Yamamoto R, Ishikura H, Azuma M, Hareyama H, Makinoda S, Koyama Y, et al. Alpha-fetoprotein production by a hepatoid adenocarcinoma of the uterus. J Clin Pathol. 1996;49:420-2.

DOI: $10.5455 / 2320-1770 . i j r \operatorname{cog} 20140952$

Cite this article as: Wani NH, Sekhon R, Giri S, Rawal SK. Hepatoid carcinoma of ovary: a very rare histopathological finding. Int J Reprod Contracept Obstet Gynecol 2014;3:824-6. 